\title{
Frequency and long term follow up of valvar insufficiency caused by retrograde aortic radiofrequency catheter ablation procedures
}

\author{
A Olsson, B Darpö, L Bergfeldt, M Rosenqvist
}

\begin{abstract}
Objective-To assess the frequency of valvar complications caused by left sided radiofrequency catheter ablation using the retrograde aortic technique.

Methods-179 patients (118 male) with a mean (SD) age of 43 (17) years underwent 216 procedures at one centre. The target of the ablation was an accessory atrioventricular pathway in 144 patients, the atrioventricular junction in 29 patients, and a ventricular tachycardia in six patients. In 25 patients structural heart disease was identified before the procedure (ischaemic heart disease 10, cardiomyopathy nine, valvar three, other three). Echol Doppler examinations were performed the day before the procedure and within 24 hours postablation; the investigations were all reviewed by the same investigator. Patients with identified valvar injury caused by the procedure were followed for 42 (7) months.
\end{abstract}

Results-Valvar injury caused by the ablation procedure was identified in four young (age 30 (8) years), otherwise healthy patients with left lateral atrioventricular accessory pathways. Mild mitral insufficiency with a central regurgitation jet was detected in two patients and remained unchanged at follow up. Mild aortic insufficiency was detected in another two patients. In one of these the regurgitation jet was central and remained unchanged at follow up. In one patient the regurgitation jet was located between the noncoronary and left cusps in relation to a loosely attached structure. Both the structure and the valvar regurgitation disappeared during follow up. No clinical complications occurred in any of the patients during follow up.

Conclusion-In this study, the frequency of valvar complications after left sided radiofrequency catheter ablation using the retrograde aortic technique was $1.9 \%$. (Heart 1999;81:292-296)

Keywords: radiofrequency catheter ablation; valvar disorders; retrograde aortic

Radiofrequency catheter ablation is a well established method for the treatment of supraventricular re-entrant tachycardias and certain forms of ventricular tachycardias. ${ }^{1-6}$ For patients with drug refractory atrial fibrillation, catheter ablation of the atrioventricular junc- tion with subsequent pacemaker implantation often improves quality of life, ${ }^{7-10}$ as well as left ventricular performance. ${ }^{11}$ Catheter ablation may be undertaken with a low risk of serious complications. ${ }^{1213}$ Injury to valvar structures has occasionally been described, ${ }^{3-15}$ but previous studies have shown divergent frequency of this complication. ${ }^{16-18}$ The studies have either been confined to children and adolescents, ${ }^{18}$ not included consecutive cases, ${ }^{17}$ or have been carried out at several centres by different investigators. ${ }^{16}$ The frequency of valvar lesions has been as high as $12 \%$ for the mitral valve and $30 \%$ for the aortic valve. ${ }^{18}$ We therefore undertook a study to assess the frequency of valvar lesions caused by retrograde aortic radiofrequency catheter ablation among a large number of consecutive patients who underwent the procedure at one centre. To diminish interobserver variability the same experienced echocardiographer reviewed all echo studies. Patients with identified valvar lesions were followed for a prolonged period in order to study whether progress or complications related to the valvar lesions would occur.

\section{Methods}

PATIENTS

One hundred and seventy nine patients underwent a total of 216 ablation procedures, which all included passage of the aortic valve in the retrograde direction with the ablation catheter. There were 118 men and 61 women in the study with a mean (SD) age of 43 (17) years, ranging from 11 to 80 years. The clinical characteristics of all patients are shown in table 1 . There were 144 patients with the WolffParkinson-White (WPW) syndrome; 136 had a single pathway and the remaining eight patients had two pathways (in only one patient were both pathways ablated from the left side). The location of the pathways that were approached from the left side were left lateral in 102 patients, left posterior in 24, posteroseptal in 15 , midseptal in two, and in one patient left lateral and left posteroseptal. Sixty nine of the pathways were concealed. One hundred and sixteen of the WPW patients underwent one ablation procedure, 21 patients two procedures, five patients three procedures, and two patients underwent four ablation procedures.

Twenty nine patients underwent left sided ablation of the atrioventricular junction when a right sided approach had failed. ${ }^{19}$ Among these 29 patients, 10 had chronic and 18 paroxysmal atrial fibrillation, and in one patient an ectopic atrial tachycardia was the underlying arrhythmia (table 1). 
Table 1 Clinical characteristics of patients

\begin{tabular}{lllll}
\hline & WPW & AVf & $V T$ & Total \\
\hline $\begin{array}{l}\text { Patients (n) } \\
\text { Radiofrequency procedures (n) }\end{array}$ & 144 & 29 & 6 & 179 \\
Male/female (n) & 181 & 29 & 6 & 216 \\
Mean (SD) age (years) & $97 / 47$ & $17 / 12$ & $5 / 1$ & $118 / 61$ \\
Structural heart disease (n) & $38(15)$ & $63(11)$ & $46(15)$ & $43(17)$ \\
$\quad$ None & 137 & 12 & 5 & 154 \\
$\quad$ Ischaemic & 1 & 8 & 1 & 10 \\
Cardiomyopathy & 2 & 7 & 0 & 9 \\
Valvar & 3 & 0 & 0 & 3 \\
Other & 1 & 2 & 0 & 3 \\
Mean (SD) arrhythmia duration (years) & $14(12)$ & $7(5)$ & $10(10)$ & $13(11)$ \\
Mean (SD) antiarrhythmic drugs tried & $1.1(0.8)$ & $1.8(0.9)$ & $2.2(0.8)$ & $1.3(0.9)$ \\
Mean (SD) radiofrequency applications & $9(9)$ & $12(7)$ & $8(6)$ & $10(9)$ \\
Mean (SD) fluoroscopy time (min) & $58(36)$ & $53(18)$ & $61(39)$ & $58(34)$ \\
Mean (SD) procedure time (min) & $208(70)$ & $158(41)$ & $229(76)$ & $202(69)$ \\
\hline
\end{tabular}

WPW, Wolff-Parkinson-White syndrome; AVJ, atrioventricular junctional ablation; VT, ventricular tachycardia.

Six patients with ventricular tachycardias originating from the left ventricle underwent one procedure each. In five of the patients the tachycardia was "fascicular" in origin and no concomitant heart disease could be identified.

\section{ABLATION PROCEDURE}

The ablation procedure was performed in the fasting and sedated state. Antiarrhythmic drugs were discontinued for at least five half lives, except for the patients who underwent ablation of the atrioventricular junction in whom the medication was maintained. A quadripolar catheter was positioned in the right ventricle and, in patients with WPW syndrome, an octapolar catheter was positioned in the coronary sinus. The ablation was performed using a $4 \mathrm{~mm}$ tip steerable, radiofrequency ablation catheter. Until 1994, the ablation was performed without temperature control and thereafter mainly with temperature control $(\mathrm{n}=77)$. The generators used were Radionics RFG-3B (Radionics Inc, Burlington, Massachusetts, USA), Medtronic Atakr (Medtronic Inc, Minneapolis, USA) and EPT 1000 (Boston Scientific Corp, Natick, Massachusetts, USA). The ablation catheters used were Polaris (without temperature control, $\mathrm{n}=139$, Mansfield, Boston Scientific Corp), Marinr MC (with thermocouple, $\mathrm{n}=58$, Medtronic Inc), and EPT Steerocath-T (with thermistor, $\mathrm{n}=19$, Boston Scientific Corp). The procedures were performed as previously described..$^{12}$ The aortic valve was passed using uniplane fluoroscopy, with the catheter bent in a J shape, as looked upon from a frontal view. The handling of the catheter in the

Table 2 Patients with valvar regurgitation detected after the radiofrequency procedure

\begin{tabular}{lllll}
\hline & Patient 1 & Patient 2 & Patient 3 & Patient 4 \\
\hline Diagnosis & WPW & cWPW & WPW & cWPW \\
Age (years) & 27 & 42 & 28 & 23 \\
Sex & Female & Female & Male & Male \\
Heart disease & 0 & 0 & 0 & 0 \\
AP loc & LL & LL & LL & LL \\
RF & $1 / 1$ & $2 / 3$ & $1 / 1$ & $1 / 1$ \\
Appl (n) & 16 & 61 & 2 & 7 \\
Temp & 0 & 0 & 1 & 0 \\
Catheter & Polaris & Polaris & Marinr MC & Polaris \\
Valve & Mitral & Mitral & Aortic & Aortic \\
Follow up (months) & 40 & 46 & 33 & 49 \\
\hline
\end{tabular}

(c)WPW, (concealed) Wolff-Parkinson-White syndrome; AP loc, localisation of the atrioventricular accessory pathway; RF, radiofrequency procedure that caused the valvar damage; LL, left lateral; Appl (n), number of applications of radiofrequency energy; Temp, use of temperature monitoring radiofrequency system. supra-aortic position was at the discretion of the performing physician, and most often included pushing the catheter carefully against the sinus of Valsalva to create the desired bend. During the last year, the technique was sometimes adjusted, using the catheter's bending capability to form the bend before reaching the aortic root. The exact handling of the catheter and the number of valvar passages were not noted in the protocol.

The patients who underwent ablation of the atrioventricular junction received a permanent pacemaker either before or within 24 hours after the ablation procedure and stayed in hospital for two to three days. The remaining patients were routinely discharged the following day.

\section{ECHOCARDIOGRAPHIC EXAMINATION}

All patients underwent echocardiographic and Doppler examination before the procedure. In the vast majority of the patients this was performed the day before the procedure, otherwise within the preceding month. The examination was repeated the day after the ablation procedure. The transthoracic two dimensional/Doppler studies were performed by experienced technicians, using either an Acuson 128 XP (Mountain View, California, USA) or a Vingmed CFM 800 (Vingmed AB, Horten, Norway). Parasternal long and short axis, apical two and four chamber views were obtained. The wall thickness, chamber size, left atrial size, and aortic root diameter were calculated in a standard manner from parasternal views. Colour Doppler, pulsed wave Doppler, and continuous wave Doppler were used to detect and grade valvar regurgitation. All studies were recorded on videotape and the results of the pre- and postablation studies were compared, regarding mitral and aortic valve dysfunction, by one experienced echocardiographer. The degree of mitral and aortic regurgitation was estimated by comparing the intensity between the antegrade and retrograde flow with continuous wave Doppler, by studying the jet width and area with colour Doppler, and by registering maximal antegrade flow velocities. Regurgitation was graded as mild, moderate, moderately severe, or severe. ${ }^{2021}$

\section{Results}

In four patients (patients 1-4), valvar insufficiencies were detected which had developed in relation to the radiofrequency procedure (fig 1). The clinical characteristics and data from the procedures are presented in table 2 . All patients were healthy without concomitant heart disease and they were all treated because of atrioventricular reciprocating tachycardias. The procedures were uneventful and the valvar lesions were detected at routine examination on the following day. In one patient, in whom the procedure was unsuccessfully performed in 1993 , the procedure was markedly prolonged and involved 61 radiofrequency applications. All of the applications were less than 20 seconds in duration and 57 lasted $\leqslant 10$ seconds. This patient later underwent a successful ablation of the pathway using four 
A

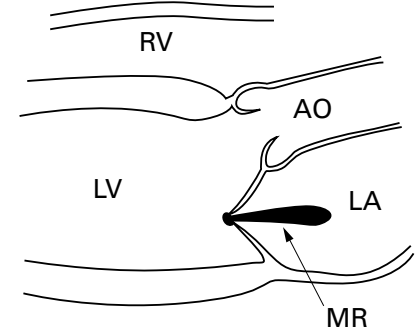

B

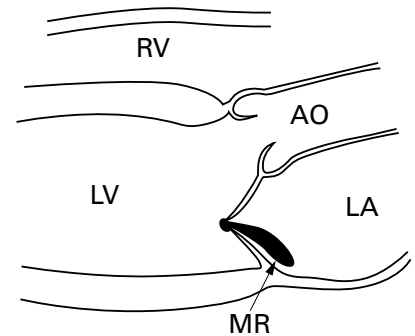

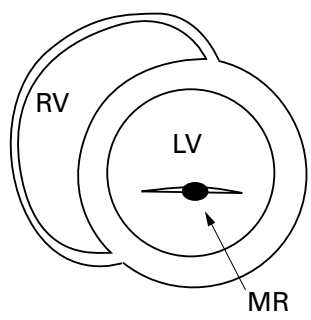

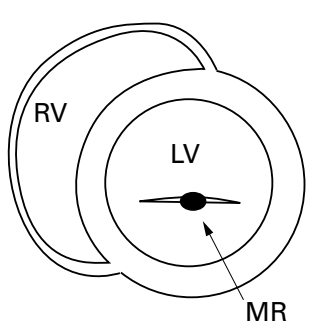

$\mathrm{C}$

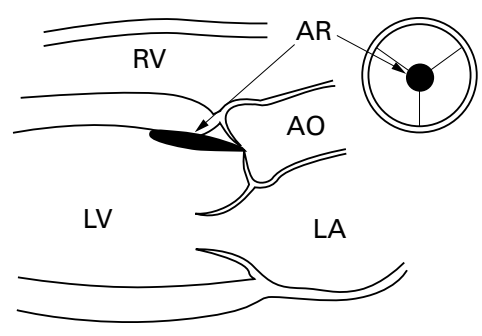

D
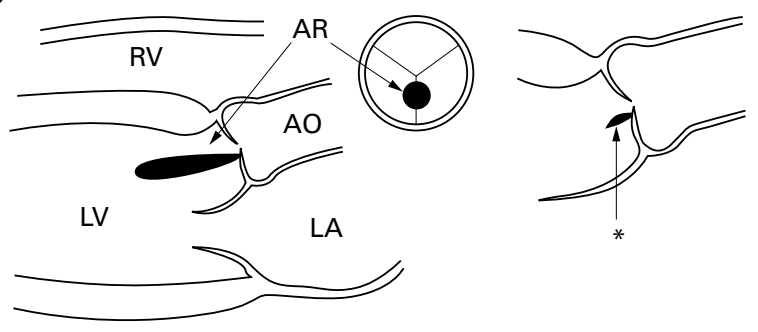

Figure 1 Schematic diagrams of parasternal long axis and short axis projections. (A) Patient 1: mild, central mitral regurgitation; normal valve leaflets and dimensions; unchanged findings at follow up. (B) Patient 2: mild, central mitral regurgitation with the jet directed posteriorly; normal valve leaflets without detectable prolapse; normal dimensions; unchanged findings at follow up. (C) Patient 3: mild, central aortic regurgitation with an anteriorly directed jet; normal aortic cusps; normal dimensions; unchanged findings at follow up. (D) Patient 4: Mild aortic regurgitation localised between the non-coronary and the left aortic cusps; cusps were normal but a small structure was loosely attached to either cusp ( $\left.{ }^{\star}\right)$; normal dimensions. An examination one month after the procedure disclosed identical findings. After 49 months the attached structure had disappeared and the regurgitation was no longer detected. AO, aorta; $A R$, aortic regurgitation; $L A$, left atrium; $L V$, left ventricle; $M R$, mitral regurgitation; RV, right ventricle.

applications with a temperature monitoring radiofrequency catheter. In two patients the valvar insufficiency was mitral and in two aortic, and they were all graded as mild (fig 1). The physicians who performed the procedures after which the valvar damages were detected, were regarded as experienced at the time. It is of note, however, that three of the four procedures were performed early in our experience, within the first 150 ablation procedures performed at our centre.

These four patients have undergone follow up echo/Doppler examinations 33, 40, 45, and 49 months after the procedure. The valvar insufficiencies remained unchanged in three patients without progression or other signs of haemodynamic impairment. One patient (patient 4 in table 2 and fig 1D), in whom the postablation examination showed a small structure, which was attached to the aortic non-coronary or left valve leaflet, and a mild aortic regurgitation, underwent echo/Doppler examination after one and 49 months. The one month examination was identical to the one performed on the day following the ablation. The structure was no longer visible after 49 months and there was no detectable aortic regurgitation. None of the patients had experienced any clinical symptoms related to the valvar lesions and remained free from tachycardias.

A comparison between the pre- and postablation echo/Doppler examinations could be performed in 210 of 216 radiofrequency procedures $(97 \%)$. In six procedures $(3 \%)$ the comparison could not be made. This was because of non-retrievable preablation examination and significant heart disease with valvar abnormalities detected postablation in two cases, which made an adequate comparison impossible. Although transcripts of the preablation protocol confirmed a similar amount of valvar insufficiency, these patients were excluded from the comparison since a prerequisite for the study was that the same investigator should examine all echo/Doppler videotapes. In four patients the postablation videotape was not possible to evaluate and another examination was not performed because of either the death of the patient $(n=3)$ or the patient's unwillingness to undergo another examination ( $\mathrm{n}=1$, after a second procedure). The deaths all occurred in patients who had undergone atrioventricular junctional ablation and were related to severe heart disease in all cases and not to the procedure. ${ }^{22}$

The echocardiographic/Doppler examination showed only small, physiological, or no valvar insufficiencies before the radiofrequency procedure in 152 patients. ${ }^{23}$ In 27 patients significant mitral or aortic valvar disease was noted. The mitral regurgitation was graded as mild in 11 patients, moderate in seven, and moderately severe in one patient. Aortic regurgitation was found in four patients (mild in two, moderate in two). In four patients, combined valvar disease was found (mild mitral and mild aortic regurgitation in one; moderate mitral and mild aortic regurgitation in one; mild mitral and moderately severe aortic regurgitation and a moderate aortic stenosis in one; and mild regurgitation and stenosis in both the mitral and the aortic valve in one patient).

\section{Discussion}

The present study has demonstrated an incidence of valvar damage of $1.9 \%$ (95\% confidence interval (CI) 0.1 to $3.7 \%$ ) during left 
sided radiofrequency ablation procedures using retrograde aortic access to the left ventricle. The patient group consisted of consecutive cases who were examined with transthoracic two dimensional/Doppler echocardiography. All the investigations were evaluated by the same experienced echocardiographer, who also performed the follow up studies. The design of the study therefore allows us to conclude that these valvar insufficiencies were caused by the radiofrequency procedure, although it was not possible to identify the underlying mechanism.

In one patient, the echo/Doppler examination the day after the procedure disclosed a small structure which was attached to the aortic valve and seemingly caused a mild regurgitation. The same structure and degree of regurgitation was found one month later, but at the late follow up, after 49 months, the structure was no longer seen and there was no detectable regurgitation. The exact nature of this structure remains obviously unknown, but the time course of its development and later disappearance suggests that it may have been a coagulum formed on a minor valvar lesion.

\section{AORTIC VALVE INJURY}

The aortic valve may be injured either directly by the catheter tip when it is passed through the valve, or by compression and stretching of the cusps by the catheter shaft during its prolonged placement in the left ventricle. ${ }^{18} 24$ With older radiofrequency systems, without temperature control, the catheter often had to be withdrawn and wiped off because of coagulum formation on the tip. This and less effective energy applications resulted in more passages of the aortic valve and longer procedures. In this study, both patients who developed aortic insufficiency underwent quite short procedures, with two and seven energy applications, respectively, and the number of aortic passages was therefore low. Individual susceptibility may be of decisive importance, ${ }^{15}$ although the anatomy of the valvar leaflets and the dimensions of the left ventricle were normal in our two patients. Occasional cases of aortic insufficiency caused by radiofrequency procedures have been reported in earlier studies, ${ }^{325}$ and the risk seems to be higher in children and adolescents. In a study of 44 patients aged 2 to 22 years, of whom 30 had accessory pathways which were ablated using the retrograde aortic technique, there were nine cases $(30 \%, 95 \%$ CI 15 to $49 \%$ ) of newly developed mild aortic insufficiency. ${ }^{18}$ In an echocardiographic study of an adult population that consisted of 59 patients with left sided accessory pathways, the frequency was lower $(3.4 \%, 95 \%$ CI 0.4 to $12 \%) .{ }^{17}$ Although the susceptibility of the valvar leaflets may be higher in younger individuals, this may also indicate that a smaller diameter of the outflow tract and the left ventricle makes valvar injury more likely to occur. All patients in this study, in whom a valvar injury was observed, had left lateral accessory pathways. This localisation is the most remote from the outflow tract, and the compression of the leaflets may therefore be augmented during the manoeuvres of the catheter to reach this position.

MITRAL VALVE INJURY

Regarding the mitral valve, the tissue lesions caused by the radiofrequency energy may directly injure the valvar structure. The lesions are placed at the base of the leaflets, and may cause valvar insufficiency if a leaflet or a chord is trapped underneath the tip of the catheter. Both of the patients with newly developed mitral insufficiency had undergone prolonged procedures with a high number of energy applications, which may have contributed to the injuries. In the paediatric study by Minich, ${ }^{18}$ the incidence of mitral insufficiency was $13 \%$ (CI 4 to $31 \%$ ) and the injury was unrelated to the number of energy applications. In a prospective, multicentre study performed by the Atakr investigators group, the same incidence was $9 \%$ (CI 4 to $15 \% ; 10$ cases among 109 patients with left sided accessory connections). ${ }^{16}$ These authors found it difficult to relate the valvar injury to the target of the ablation, since 11 of 21 patients with newly developed mitral insufficiency had neither undergone transmitral ablation nor ablation inside the left ventricle.

Altogether, these data indicate that there is a small but definite risk of valvar injury of both the aortic and mitral valves during radiofrequency procedures using the retrograde aortic approach.

FOLLOW UP ECHOCARDIOGRAPHY

During a follow up period of 33 to 49 months, the degree of regurgitation as well as all echocardiographic measurements remained unchanged in three individuals, and no clinical complications related to the valvar injury were observed. Obviously, since the patients in whom the valvar complication occurred are quite young, this follow up time is much too short to give an estimate of the long term risk in the life long perspective. Although the clinical significance of these valvar injuries is unknown, we assume that the risk of endocarditis is similar for these patients as for other patients with minor valvar abnormalities.

CLINICAL IMPLICATIONS

Although not definitely proven, it seems reasonable to suggest that the risk of valvar injuries when using retrograde aortic approach is in direct relation to the duration of the procedure and the number of energy applications and passages of the aortic valve. The use of modern radiofrequency equipment with temperature control makes for shorter procedures, but the operator's skill and experience is equally important. A continued refinement of the ablation catheters will lead to the development of more flexible and better performing catheters and the use of small diameter catheters may be less harmful for the valves, especially in children. Despite these future perspectives, a small risk of sustained valvar injuries should be considered when giving advice about whether to treat a minimally symptomatic patient. In patients with valvar 
lesions caused by catheter ablation, prophylactic use of antibiotics should be advocated in conjunction with surgery and minor urogenital tract and dental procedures.

\section{LIMITATIONS}

Six patients in this study were excluded because of insufficient pre- or postablation echocardiographic examinations. Five of these patients had significant heart disease. We have no reason to believe that the valvar abnormalities were caused by the procedure, since the same degree of regurgitation was described in the written reports from the insufficient examinations. The patients were excluded, however, which may have led to an underestimation of the incidence of valvar injury following the procedures.

We would like to express our gratitude towards Margareta Arfss, $\mathrm{RN}$, and Mats Andersson, RN, for excellent assistance in data collection and study monitoring. This study was supported by a grant from the Swedish Heart and Lung Foundation.

1 Jackman W, Wang X, Friday KJ, et al. Catheter ablation of accessory atrioventricular pathways (Wolff-ParkinsonWhite syndrome) by radiofrequency current. $N$ Engl f Med 1991;324:1605-11.

2 Morady F, Harvey M, Kalbfleish S, et al. Radiofrequency catheter ablation of ventricular tachycardia in patients with coronary artery disease. Circulation 1993;87:363-72.

3 Calkins H, Langberg JJ, Sousa J, et al. Radiofrequency catheter ablation of accessory atrioventricular connections in 250 patients. Abbreviated therapeutic approach to WolffParkinson-White syndrome. Circulation 1992;85:1337-46.

4 Langberg J, Sousa J, El-Atassi R, et al. Radiofrequency catheter ablation of accessory atrioventricular connections in 250 patients. Circulation 1992;85:1337-46.

5 Coggins DL, Lee RJ, Sweeney J, et al. Radiofrequency catheter ablation as a cure for idiopathic tachycardia of both left eter ablation as a cure for idiopathic tachycardia of both left and right $1333-41$.

6 Nakagawa H, Beckman KJ, McClelland JH, et al. Radiofrequency catheter ablation of idiopathic left ventricular tachycardia guided by a Purkinje potential. Circulation 1993;88:2607-17

7 Langberg J, Chin M, Rosenqvist M, et al. Catheter ablation of the atrioventricular junction with radiofrequency energy. Circulation 1989;80:1527-35.

8 Fitzpatrick A, Kourouyan H, Siu A, et al. Quality of life and outcomes after radiofrequency His-bundle catheter ablation and permanent pacemaker implantation: impact of treatment in paroxysmal and established atrial fibrillation. Am Heart f 1996;131:499-507. $9 \begin{aligned} & \text { Jensen J, Bergfeldt L, Rosenqvist } M \text {. Long-term follow-up of } \\ & \text { patients treated by radiofrequency ablation of the atrioven- }\end{aligned}$ patients treated by radiofrequency ablation of the atrioven-
tricular junction. Pacing Clin Electrophysiol 1995;18:160914.

10 Brignole M, Gianfranchi L, Menozzi C, et al. Assessment of atrioventricular junction ablation and DDDR modeswitching pacemaker versus pharmacological treatment in patients with severely symptomatic paroxysmal atrial fibrillation. Circulation 1997;96:2617-24.

11 Edner M, Caidahl K, Bergfeldt L, et al. Prospective study of left ventricular function after radiofrequency ablation of atrioventricular junction in patients with atrial fibrillation. Br Heart f 1995;74:261-7.

12 Kay GN, Epstein AE, Dailey SM, et al. Role of radiofrequency ablation in the management of supraventricular arrhythmias. If Cardiovasc Electrophysiol 1993;4: 371-89.

13 Greene TO, Huang SK, Wagshal AB, et al. Cardiovascular complications after radiofrequency ablation of supraventricular tachyarrhythmias. Am f Cardiol 1994;74:615-17.

14 Conti JB, Geiser E, Curtis AB. Catheter entrapment in the mitral valve apparatus during radiofrequency ablation. Pacing Clin Electrophysiol 1994;17:1681-5.

15 Seifert M, Morady F, Calkins H, et al. Aortic leaflet perforation during radiofrequency ablation. Pacing Clin Electrophysiol 1991;14:1582-5.

16 Pires LA, Huang SK, Wagshal AB, et al. Clinical utility of routine transthoracic echocardiographic studies after uncomplicated radiofrequency catheter ablation: a prospective multicenter study. The Atakr investigators group. Pacing Clin Electrophysiol 1997;19:1502-7.

17 Neuzner J, Faude I, Pitschner $\mathrm{HF}$, et al. Incidence of intervention-related heart valve lesions after highfrequency catheter ablation of left-sided accessory atrioventricular connections [in German]. Z Kardiol 1995;84: 1002-8.

18 Minich LL, Snider RA, Dick M. Doppler detection of valvular regurgitation after radiofrequency ablation of accessory connections. Am f Cardiol 1992;70:116-17.

19 Sousa J, El-Atassi R, Rosenheck S, et al. Radiofrequency catheter ablation of the atrioventricular junction from the left ventricle. Circulation 1991;84:567-71.

20 Perry GJ, Helmcke F, Nanda NC, et al. Evaluation of aortic insufficiency by Doppler color flow mapping. I Am Coll Cardiol 1987;9:952-9.

21 Miyatake K, Izumi S, Okamoto M, et al. Semiquantative grading of severity of mitral regurgitation by real-time twodimensional Doppler flow imaging technique. $7 \mathrm{Am}$ Coll Cardiol 1986;7:82-8.

22 Darpö B, Walfridsson $\mathrm{H}$, Aunes $\mathrm{M}$, et al. Incidence of sudden death after radiofrequency ablation of the atriovenden death after radiofrequency ablation of the atrioventricular junction

23 Yoshida K, Yoshikawa J, Shakudo Mea. Color Doppler evaluation of valvular regurgitation in normal subjects. Circulation 1988;78:840-7.

24 Lau YR, Case CL, Gilette PC, et al. Frequency of atrioventricular valve dysfunction after radiofrequency catheter ablation via the atrial approach in children. Am $\mathcal{F}$ Cardiol 1994;74:617-18.

25 Lesh MD, Van Hare G, Scheinmann M, et al. Comparison of the retrograde and transseptal methods for ablation of left free wall accessory pathways. I Am Coll Cardiol 1993;22:542-9. 